Estudios sobre armas antiguas, arte militar $\mathrm{y}$ vida cultural en oriente y occidente XXXIX (2019), pp. 7-18

ISSN: 0436-029X

https://doi.org/10.3989/gladius.2019.01

\title{
CONSIDERACIONES SOBRE LA ESPADA DE ANTENAS ENCONTRADA EN EL RIO CAVALLERA (LIGURIA, ITALIA)
}

\author{
REMARKS ON THE ANTENA-SWORD FOUND \\ IN THE CAVALLERA RIVER (LIGURIA, ITALY)
}

POR

\author{
Raimon Graells i Fabregat*, Gustau García JimÉneZ ${ }^{* *}$ y Angiolo del Lucchese***
}

\section{Resumen - ABstract}

El artículo presenta una inusual espada de antenas cuya tipología enlaza las tradiciones centro-europeas y las características del golfo de León. La espada fue depositada inutilizada en una tumba, pero fue encontrada en un curso fluvial. La singularidad de su tipología pone en evidencia el interés por el estudio de las armas en áreas culturales tan complejas y ricas como Liguria.

The article presents an unusual sword whose typology links the Central European traditions and those of the Gulf of Lion. Despite the sword was deposited bended in a grave during the Iron age, it was found in 2013 in a river course. The uniqueness of its typology highlights the interest in the study of weapons in cultural areas as complex and rich as Liguria.

\section{Palabras Clave - Keywords}

Espada de Antenas; interacción cultural; Primera Edad del Hierro; golfo de León.

Antea sword; cultural interaction; Iron Age I; Gulf of Lion.

\section{Cómo CITAR ESTE ARTículo / Citation}

Graells i Fabregat, R.; García Jiménez, G. y Del Lucchese, A. (2019): «Consideraciones sobre la espada de antenas encontrada en el rio Cavallera (Liguria, Italia)». Gladius, XXXIX: 7-18. https://doi.org/10.3989/ gladius. 2019.01

Las anomalías son siempre atractivas. Lo que sale de la norma, lo que completa un amplio abanico tipológico o lo que escapa de dicha tipología es lo que ayuda a comprender tentativas de cambio, adopciones de estímulos e interacciones particulares. Este es el caso de la inusual espada hallada en el curso del río Cavallera en la localidad ligur de Carcare (Prov. Savona, Liguria / I) y por ello llama la atención y requiere un estudio pormenorizado que ponga en valor

* Römisch-Germanisches Zentralmuseum (RGZM), Leibniz-Forschungsinstitut für Archäologie, Kompetenzbereich Vor- und Frühgeschichte. Ernst-Ludwig Platz, 2; 55116, Mainz (Deutschland), graells@rgzm.de / ORCID iD: https://orcid.org/0000-0002-9057-7510

*** Desperta Ferro - Ediciones, gust_mei@hotmail.com / ORCID iD: https://orcid.org/0000-0002-2955-1493

${ }^{* * *}$ Soprintendenza per i Beni Archeologici della Liguria, via Balbi 10, 16100, Genova (Italia), adl.sop@libero.it / ORCID iD: https://orcid.org/0000-0002-4280-1231 
sus características y permita su inclusión en el debate internacional sobre la conformación de modelos de espadas y su evolución.

El trabajo que sigue no analizará ni los motivos de su inutilización ni las causas que la llevaron al curso de agua donde se recuperó. La atención se concentra en su particular tipología híbrida, próxima en algunos detalles a los modelos de espadas de antenas recuperadas en el golfo de León occidental y, en otros particulares, a las espadas de tradición centroeuropea. Con ello se quiere evidenciar la importancia de esta pieza como bisagra entre distintas tradiciones culturales hasta hoy sin apenas testimonios, y la necesidad de conocer el catálogo de las armas ligures para seguir estudiando el intercambio de influencias y la transformación de los modelos de armas entre los grupos culturales occidentales, centroeuropeos e itálicos.

\section{EL DESCUBRIMIENTO}

La espada del río Cavallera, un afluyente del río Bormida di Carcare, se localizó en su lecho seco en otoño 2013 (Fig. 1) (Del Lucchese, Maestro y Prestipino, 2015). La zona era conocida desde hacía algún tiempo y, en consecuencia, se hallaba bajo observación por distintas

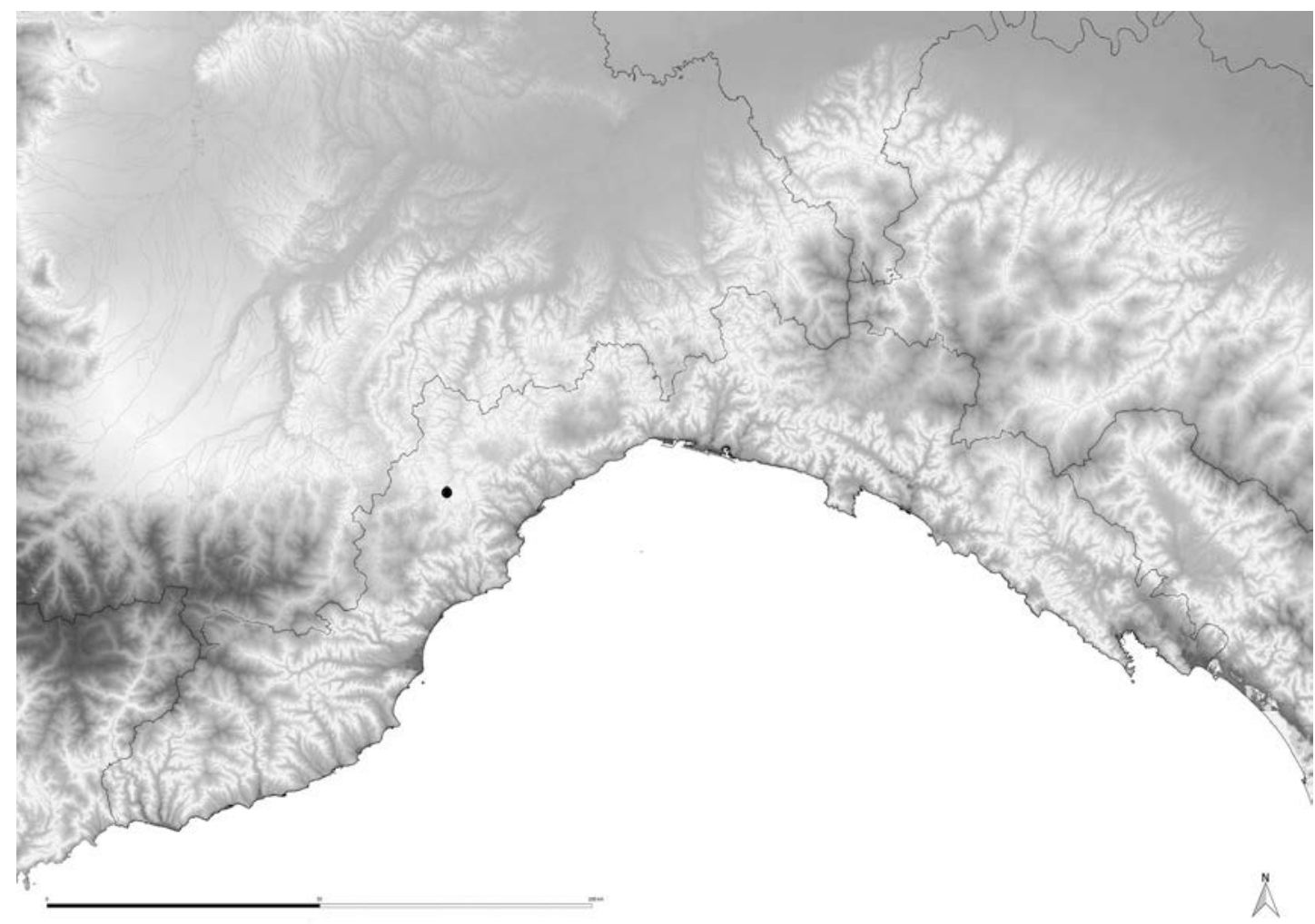

Figura 1. Situación del río Cavallera (Carcare), donde fue hallada la espada de antenas en 2013.

razones: por un lado, debido a la presencia de incisiones rupestres descubiertas por Carmelo Prestipino en 2004; por otro, porque en 2009 se recuperaron en el curso del río Cavallera un casco de hierro y una punta de lanza plegada (Del Lucchese y Prestipino, 2013). Poco después, 

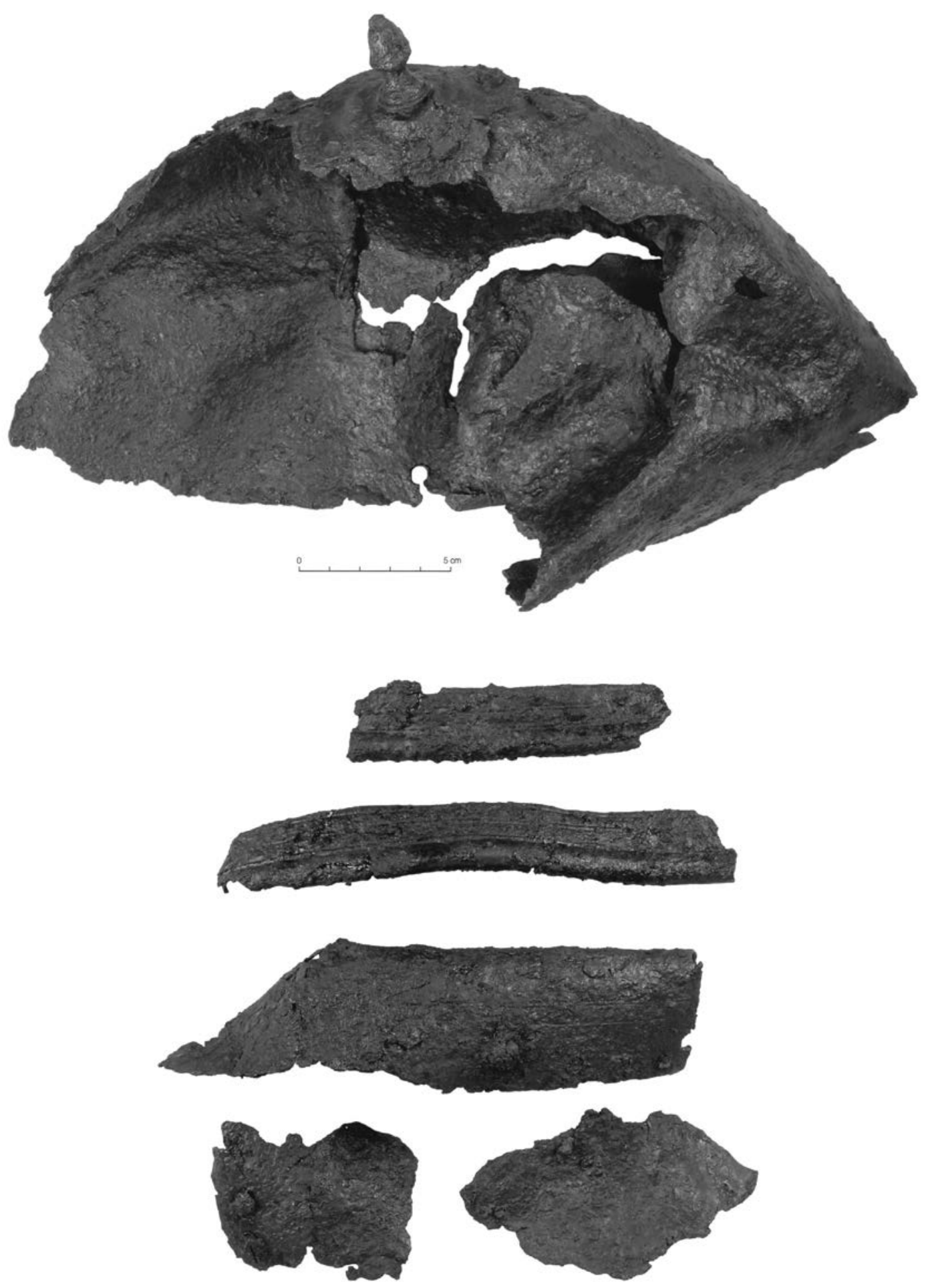

Figura 2. Casco de hierro hallado en 2009 en el río Cavallera (Carcare). Fotografía: Archivio della Soprintendenza per i Beni Archeologici della Liguria. 

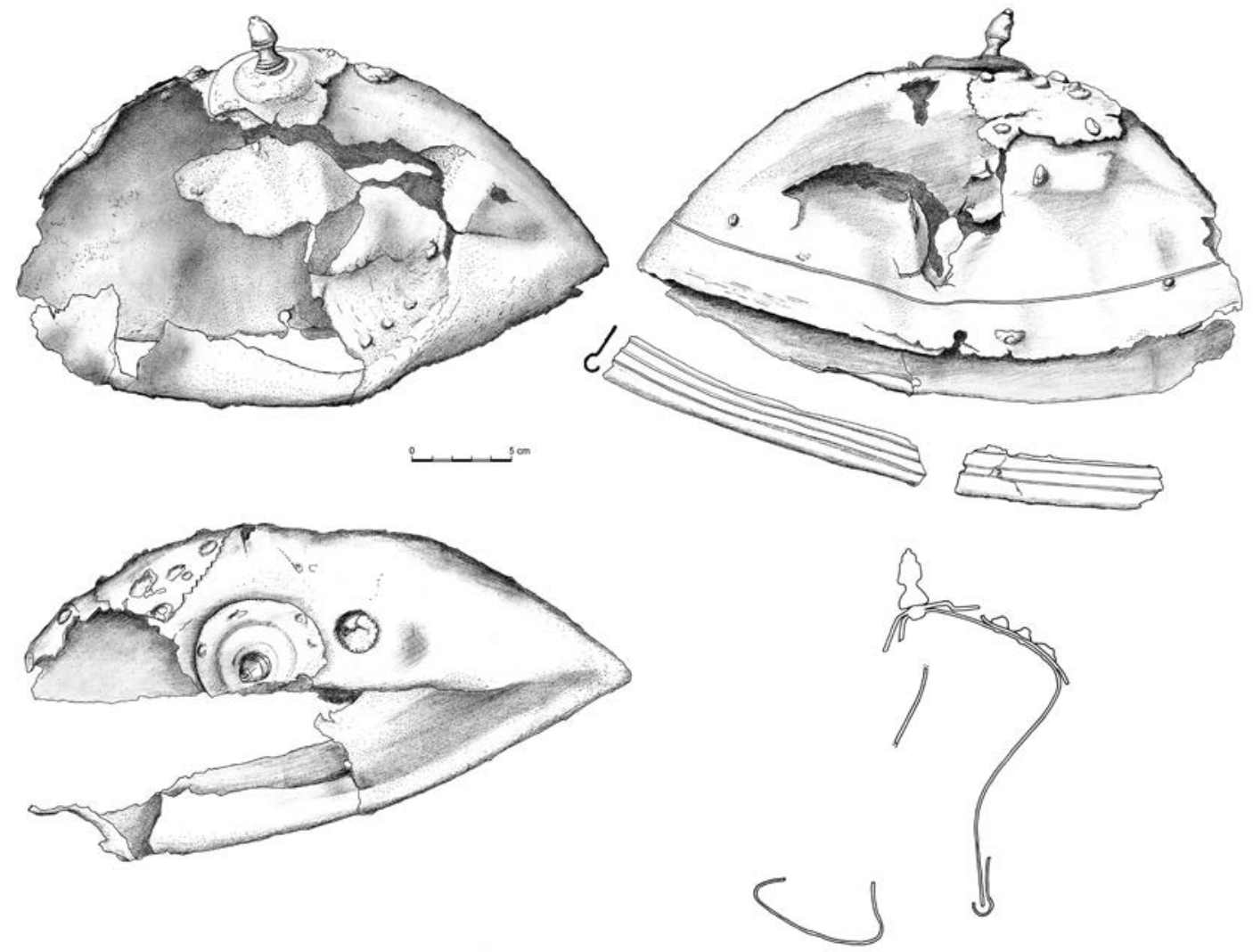

Figura 3. Casco de hierro hallado en 2009 en el río Cavallera (Carcare). Dibujo de Laura Tomasi.

en 2013, próximo al lugar de los hallazgos previamente comentados, fue encontrada la espada objeto de este trabajo.

El casco de hierro (Figs. 2a-b-3), tipológicamente próximo a los ejemplares de Varenna (Italia) y de Greifenstein (Austria) (Egg, 1992), pese a estar pendiente de un estudio detallado,
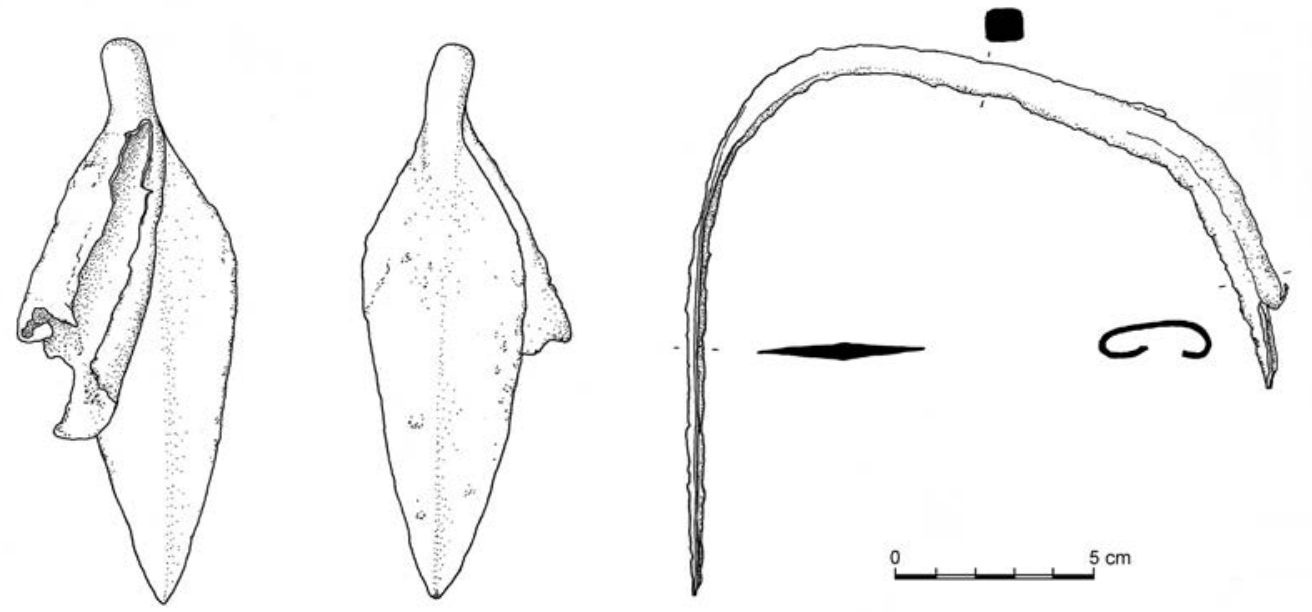

Figura 4. Punta de lanza hallada en 2009 en el río Cavallera (Carcare). Dibujo de Laura Tomasi. 
presenta características que pueden situarlo en el período La Tène A-B1: un aplique superior en forma de huso, un refuerzo aplicado al borde en hierro y una calota hemisférica. Al margen quedan dos discusiones que precisan de un estudio particular: por un lado, la vistosa reparación conseguida mediante el remachado de una placa de hierro desde el exterior; por otro, el estudio del modo como fue inutilizado. Esta inutilización, en cualquier caso, aproxima el casco a la espada que veremos a continuación, así como con la mencionada punta de lanza (Fig. 4). Estas características permitieron rápidamente interpretar el conjunto como armas pertenecientes a tumbas destruidas (en el lado sur del curso de agua, se han identificado varias áreas funerarias de la Edad del Hierro) y arrastradas por la corriente (Del Lucchese y Prestipino, 2013), descartándose la posibilidad de que fueran fruto de algún tipo de depósito de armas ofrendado a las aguas (sobre el argumento vid. Gambari, 2000; Dedet y Marchand, 2015a; Graells y Lorrio, 2013, 2016). El interés por el conjunto y por el estudio del área es objetivamente relevante, pero lo es también la falta de paralelos para la espada y la datación propuesta (mediados del s. VI a. C.) pues la distancian claramente de lo conocido en el resto del golfo de León.

\section{UNA ESPADA DE ANTENAS CON UN ÚNICO FILO (Figs. 5-7)}

Longitud máxima: $68 \mathrm{~cm}$.

Longitud de la hoja: $57,4 \mathrm{~cm}$.

Longitud de la empuñadura: $10,6 \mathrm{~cm}$.

Ancho máximo de la hoja: $3,4 \mathrm{~cm}$.

Grosor máximo del dorso de la hoja: $0,4 \mathrm{~cm}$.

Salvando las distancias con los problemas de nomenclatura habituales en este tipo de objetos, cuya distinción entre espada o puñal no es siempre fácil, y teniendo además en cuenta que la peculiar hoja de esta arma implica ciertas incertidumbres en cuanto a su uso efectivo y podría apuntar a un uso ritual en paralelo ${ }^{1}$, hablamos aquí de espada por considerar que, independientemente de su uso final, el concepto de este objeto está asociado al de las espadas coetáneas a ella y, al margen de ello, por la notable longitud de su hoja, que supera ampliamen-

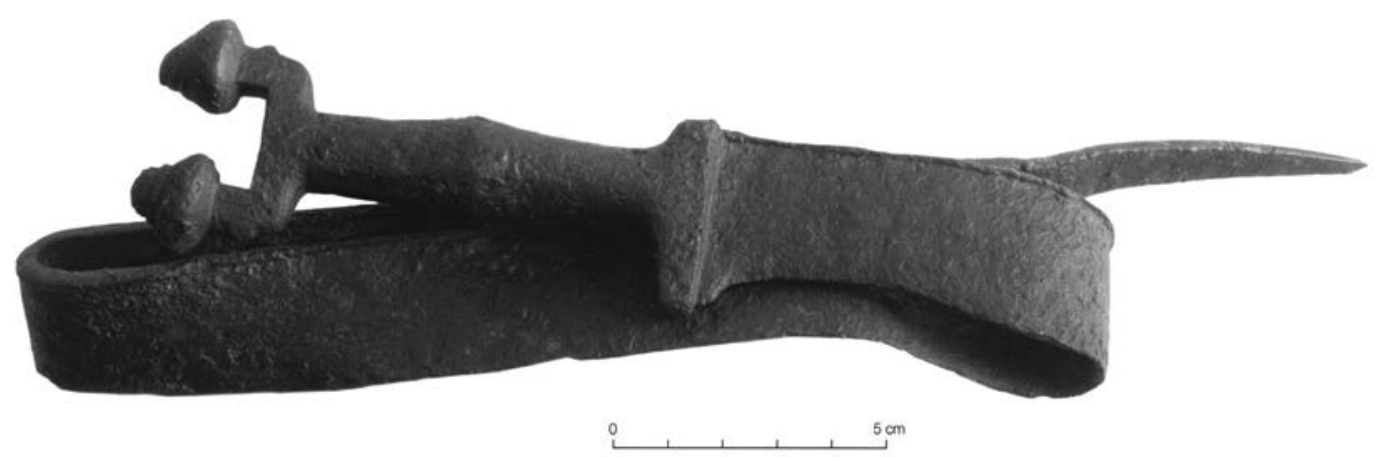

Figura 5. Espada de antenas hallada en 2013 en el río Cavallera (Carcare). Fotografía: Archivio della Soprintendenza per i Beni Archeologici della Liguria.

1 Las hojas de un solo filo en el Mediterráneo suelen estar asociadas preferentemente a cuchillos de hoja curva, habituales en tumbas y conjuntos votivos de la Primera (p. e. Beylier, 2012) y la Segunda Edad del Hierro (los ejemplos pueden ser numerosísimos solo en la península ibérica, vid. Quesada 1997: 167-168), aunque también se dan en espadas propiamente dichas, como es el caso de las falcatas (Quesada, 1997: 61-171), si bien nunca en puñales. 

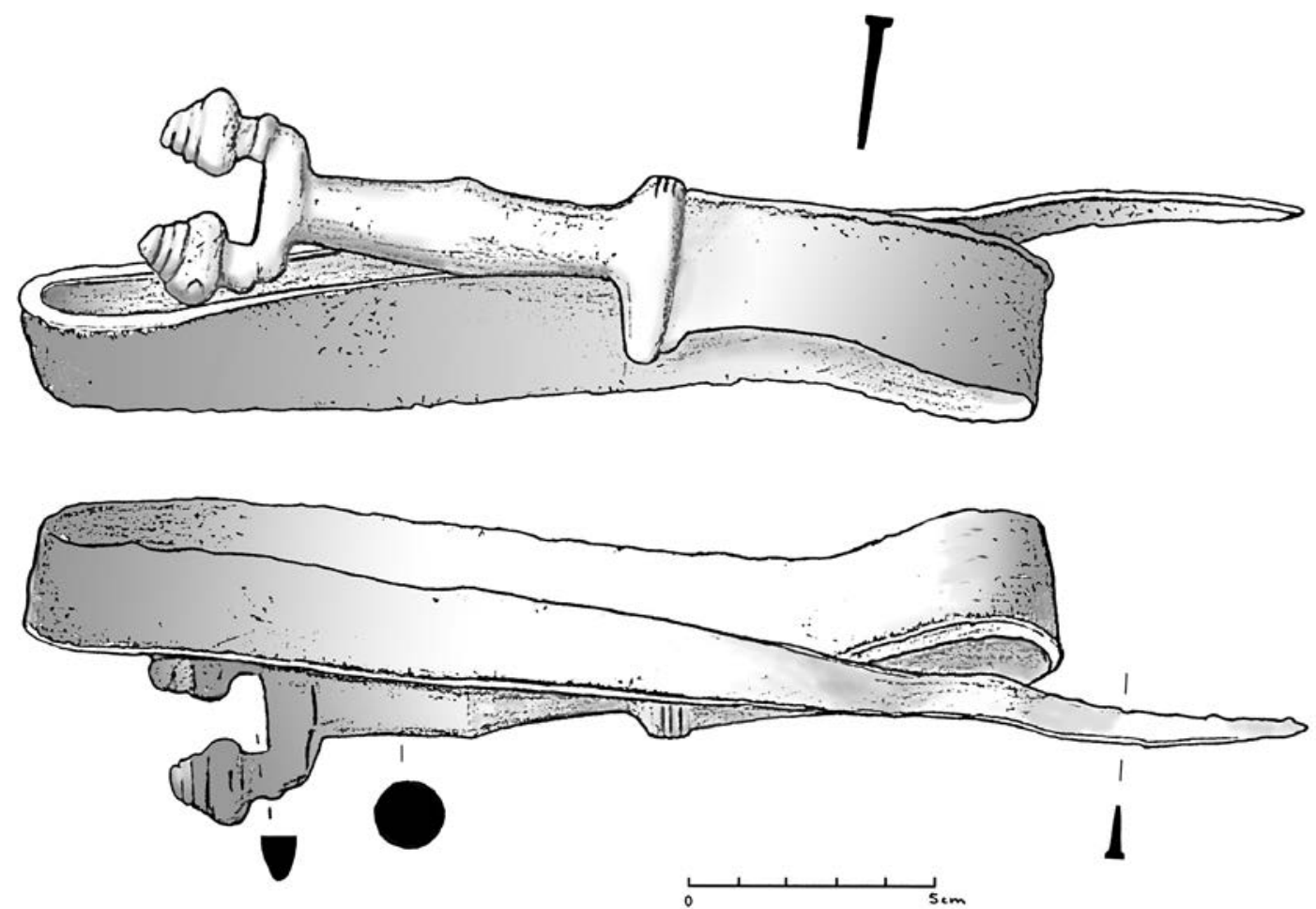

Figura 6. Espada de antenas hallada en 2013 en el río Cavallera (Carcare). Dibujo de Laura Tomasi.

te los $30 \mathrm{~cm}$ de longitud, un límite en ocasiones propuesto como mínimo indispensable para discriminar entre espadas y puñales (Beylier, 2012: 32).

Sin duda el rasgo más llamativo de este ejemplar es su peculiar hoja, de la que se pueden extraer interesantes reflexiones. Esta presenta una lámina de hierro forjado con sección triangular, y su grosor aumenta progresivamente hacia la parte dorsal, que es recta, aunque converge con suavidad hacia la punta. Dicha parte dorsal de la hoja contiene una pequeña inflexión que conforma una sección en T que resulta curiosa en comparación con ejemplares que pudieran resultar comparables. En efecto, la presencia de un único filo implica, entre otras muchas cosas, la necesaria modificación de la empuñadura para desplazar el eje de la misma hacia el lado opuesto al filo, como veremos más adelante. Este hecho repercute en un mayor control del centro de gravedad del arma, pero también supone una mayor protección de la guarda sobre la mano, evitando el riesgo de impacto con el lado sobre el que se proyectaría este durante el uso.

Pero hay otros rasgos que vale la pena destacar de la hoja: por una parte, destaca a simple vista la extrema delgadez de esta, de apenas poco más de $3 \mathrm{~cm}$, lo que unido a su considerable longitud nos ofrece un perfil netamente estilizado, acentuado por su pronunciada punta. Este hecho obliga a discutir sobre la funcionalidad de esta arma, puesto que una punta tan alargada estaría indicando una intención de uso orientada al estoque, algo que contrasta con el hecho de que el dorso tenga continuidad hasta el mismo extremo de la hoja. El otro rasgo singular es la concavidad del filo de la hoja en su parte proximal, un fenómeno poco habitual en las espadas de un solo filo conocidas hasta ahora ${ }^{2}$.

2 Un detalle que, sin embargo, se repite en otras armas de un solo filo como son las falcatas, que igualmente incluyen ese arranque más ancho en la base de la hoja antes de estrecharse en curva. Es muy probable que en la pieza de Pallare la 


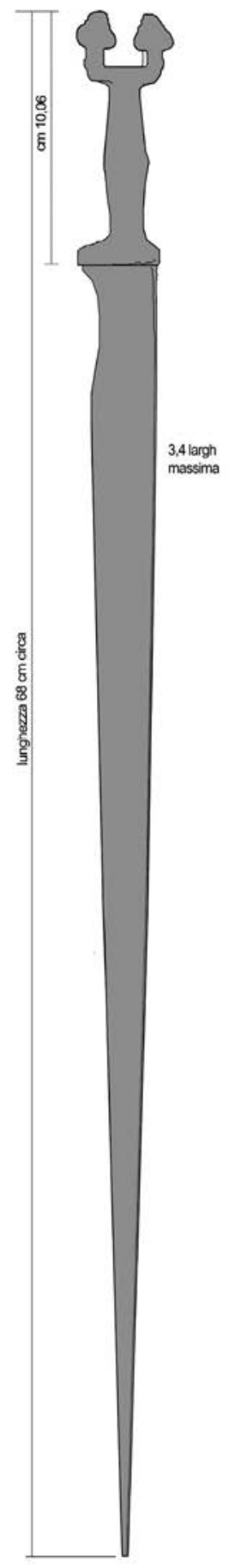

Al margen de la hoja, la conservación de la espada apenas permite hacer valoraciones en cuanto a los detalles de su empuñadura. Uno de los rasgos tipológicos más relevantes de las espadas de antenas tiene que ver con el hecho de si la hoja prolonga el alma de la empuñadura, que se encuentra oculta tras las cachas o los cilindros que conforman el mango, en forma de lengüeta, con una forma ancha y de poco grosor, o bien lo hace mediante una espiga (Farnié, 2016). La primera de estas dos fórmulas es más tardía en las armas de hierro, y solo se encuentra con cierta frecuencia en la región aquitana y suroeste de Francia (Farnié, 2016: fig. 18). A falta de radiografías de la pieza, no sabemos si la empuñadura de esta espada era de espiga o de lengüeta. Una primera impresión puede apuntar a la lengüeta si advertimos el ensanchamiento en el centro del mango, pero un examen más detallado confirma que la estructura externa de la empuñadura la conforman dos cilindros troncocónicos unidos por su parte más ancha ${ }^{3}$, un tipo de composición que acompaña habitualmente a los modelos de antenas con empuñadura de espiga posteriores al segundo cuarto del siglo VI a. C. (Farnié, 2016: 25-29).

Otra cuestión morfológica relacionada con la empuñadura son sus antenas. Estas son de forma cuadrangular y no redondeada como sería más frecuente en las regiones litorales en la mitad occidental de golfo de León durante la etapa de mediados del siglo VII hasta comienzos del siglo VI a. C. (Dhennequin, 1999: 163; Farnié, 2016: 19-22) Por otra parte, los remates de las antenas tienen una forma triangular que deriva aparentemente del añadido de varias anillas de distinto tamaño superpuestas, y no en cambio las esferas o las formas simétricas que son más habituales en las espadas de antenas de la Primera Edad del Hierro.

Asimismo, cabe mencionar la guarda de la espada, de cierto grosor y base completamente recta. Condicionada por la peculiar forma de la hoja a la que acompaña, su forma es claramente asimétrica, con una parte más desplazada del eje coincidiendo con el lado correspondiente al filo de la hoja. De nuevo, se trata de un rasgo muy singular, que nada tiene que ver con las formas semicirculares de las espadas más antiguas o las cuadrangulares de las más recientes (Farnié, 2016).

Por último, la oxidación de la pieza apenas permite distinguir mayor detalle. Sin embargo, se observan algunas estrías dispuestas en paralelo a la base de la guarda.

La espada fue cuidadosamente plegada en tres segmentos, siguiendo un procedimiento de recalentado del metal y posterior doblado y

Figura 7. Reconstrucción de la forma original de la espada original de la espada hallada en 2013 en el río Cavallera (Carcare). Dibujo de Laura Tomasi.

\footnotetext{
intención de su forma tuviera que ver con el desplazamiento del centro de gravedad lejos de la zona proximal (más cercana por tanto a la punta).

3 Sin un adecuado estudio tecnológico es complicado proponer observaciones más concretas acerca del modo como se unieron estas piezas.
} 
martilleado delicado, como lo evidencia haber evitado la formación de ángulos y fracturas. Se trata de un proceso sobradamente conocido tanto en el ámbito funerario como en el estrictamente votivo.

\section{LOS PARALELOS: ENTRE LOS MODELOS OCCIDENTALES Y LA MODA CENTROEU- ROPEA}

La tradición de espadas con antenas en el área ligur se conoce particularmente por las representaciones sobre las estelas de la Lunigiana (Paribeni, 2007; Beylier, 2012: 38-39, fig. 24), donde hasta cuatro ejemplares presentan distintos tipos de puñales o espadas rematados con empuñaduras de antenas. En todos los casos, el detalle de las representaciones parece indicar que se trata de espadas con hojas de dos filos. La espada de antenas de Pietra Ligure, único paralelo real hasta ahora recuperado en la región, presenta también una hoja con dos filos paralelos (Beylier, 2012: 402-404, pl. 336-1). En cualquier caso, está claro que las espadas de antenas no son formatos raros en la región ligur.

La tradición de espadas con un único filo tampoco es ajena al repertorio ligur. Prueba de ello son los ejemplares sin antenas de Roccatagliata (Garibaldi, 2004: 278-280, nr. IV.15.5; Del Lucchese, Maestro y Prestipino, 2015: 159) y Savignone (Garibaldi y Traverso, 2004: 280, nr. IV.16.5; Del Lucchese, Maestro y Prestipino, 2015: 159) que probablemente tengan mucho que ver con la espada de Carcare a nivel tipológico, aunque están peor conservadas. En cambio, sí es un rasgo inexistente para los modelos de espada de antenas del golfo de León ${ }^{4}$, donde ninguno de los numerosos ejemplares conocidos con empuñadura de antenas presenta un único filo ${ }^{5}$. Esta diferencia, además, se acentúa al comparar el tipo de empuñadura de la espada de Carcare con las del sector occidental del golfo de León, puesto que no presenta las habituales guardas adaptadas a la curva de los hombros de la hoja y, además, cuenta con unos remates de las antenas globulares terminados en una protuberancia decorada con círculos concéntricos.

Precisamente la decoración mediante glóbulos de las antenas se documenta de manera casi idéntica en una espada de Saint-Dalmas-de-Tende (Alpes-Maritimes / F) (Beylier 2012: 38, nr. 297, fig. 22, pl. 56F), que además resulta particular respecto a las demás por la longitud de la hoja $(54 \mathrm{~cm})$ y por la configuración de la empuñadura, aparentemente maciza y desprovista de nervio central.

La datación de la espada de Saint-Dalmas-de-Tende, que puede resultar orientativa para estimar la de la espada de Carcare, puede inducirse grosso modo a partir de su guarda de tipo envolvente, que, junto a otros indicios como el fuerte nervio decorado con incisiones longitudinales en la hoja, estaría indicando su correspondencia a la tradición de espadas de antenas de la segunda mitad del s. VI a. C. En cambio, las espadas con un único filo conocidas en Liguria, fechadas en el s. V a. C. de manera genérica, presentan filos con hojas lisas, como las del ejemplar en estudio. Esta tendencia hacia el s. V a. C. parece confirmarse al valorar entonces el tipo de inutilización de la espada, plegada de manera atenta en tres cuerpos, a diferencia de la práctica habitual en espadas de antenas del s. VI a. C. en el golfo de León (con un doblado

4 Beylier, 2012: 32-38. Tampoco hay similitudes ni paralelos claros en los rasgos de las espadas de antenas de la zona aquitana, que es la otra región donde abundan más estas producciones (vid. Mohen, 1980; Farnié, 2016: 18-31).

5 Existen cuchillos de gran tamaño del Languedoc con un único filo, que son poco habituales y cuentan con hojas muy anchas, como por ejemplo alguno de los ejemplares del depósito de Auzet (Beylier, 2012: 161, fig. 142, 2), fechado en la segunda mitad del siglo VI a. C. 
circular o en U). Inutilizaciones como las de la espada de Carcare se documentan a partir de mediados del s. V a. C. tanto para espadas como para lanzas en distintos territorios.

Merece también la pena detenerse un momento en relación a la delgadez de la hoja y la forma pronunciadamente apuntada de la misma. Revisando las hojas de espada de la fase final de la Primera Edad del Hierro en el occidente europeo ${ }^{6}$, tan solo las llamadas espadas de esferas, fechadas a finales del Hallstatt D, en torno a comienzos del siglo V a. C. (Beylier, 2012: 5054), cuentan con hojas tan estilizadas y claramente orientadas a su uso como estoque, aunque considerablemente más largas y siempre con hojas de dos filos, por lo que solo podría pensarse en una relación tipológica muy remota, en caso de que esta hubiera existido.

En cambio, formas parecidas en la hoja (salvando el detalle de la curvatura pronunciada en la parte proximal de la hoja y el ligero ensanchamiento en el centro) pero diametralmente opuestas en la empuñadura, se observan también al otro lado de la geografía septentrional itálica, en las espadas de un solo filo del norte del Adriático, y en especial en las de la región balcánica, fechadas en el siglo VI a. C. y la fase reciente del Hallstatt (ca. 500-450 a. C.). Las proporciones en las hojas de algunas de sus variantes ${ }^{7}$ se mueven entre los $3 \mathrm{~cm}$ y poco más de anchura y los $60 \mathrm{~cm}$ de longitud, un rango que coincide a la perfección con la espada de Carcare. Asimismo, la sección del dorso suele contar también con esa característica forma de T, mientras que las puntas de las hojas son igualmente largas. Resulta quizá sintomático, además, que en estos ejemplares con empuñadura lateral cuenten con guardas que forzosamente son asimétricas; esto es, con uno de sus lados, el correspondiente al del filo, más pronunciado y desbordado. Evidentemente, no se postula aquí una dependencia directa de este tipo de armas, sino más bien la apertura a un espectro de formas e influencias muy variable que pudo tener que ver en la hibridación de rasgos finalmente plasmados en la espada ligur.

Cronológicamente, pues, la combinación de observaciones lleva a fechar la espada de Carcare entre finales del s. VI y mediados del s. V a. C., fecha más acorde con la cronología esperada si seguimos relacionando el lugar del hallazgo y los materiales previamente allí recuperados como parte de un área funeraria destruida y, en particular, en relación con la datación del casco de hierro ${ }^{8}$.

Por otra parte, mientras para el golfo de León este tipo de espadas con un único filo parece una anomalía, en Centroeuropa se observa como los mismos tipos de espadas o puñales de antenas pueden presentar uno o dos filos de manera coetánea sin que eso implique distinciones espaciales o de talleres ${ }^{9}$. Ignorando por ahora la presencia de formatos similares en un número importante para la región ligur, es verosímil pensar en una moda particular según la cual el portador decidiría a partir de sus preferencias personales, pese a que esto implique modos de combate distintos determinados por la morfología de las hojas de las espadas. No obstante, no hay que descartar que la peculiar fórmula tipológica escogida en esta espada, con detalles que sintetizan influencias occidentales, centroeuropeas y quizá también balcánicas, no esté orientada a su vez, aunque fuera a nivel simbólico, a un papel relacionado con lo ritual. En cualquier caso, la recepción de esta moda centroeuropea (o de la parte oriental del norte de Italia) indica la transformación de estos influjos en el área ligur.

6 A comienzos de la Primera Edad del Hierro son en cambio más frecuentes, puesto que en esta fase el trabajo del hierro es todavía poco preciso y no es raro que se den fórmulas experimentales que tiendan a exagerar la longitud de las hojas y su aspecto más delgado (Farnié, 2016: 19-24).

7 Por ejemplo, Gaspari y Mlinar, 2005, en particular entre la variante de Trzisze-Donja Dolina y, sobre todo, la variante Smihel, que es evolución de aquella; también en Quesada, 1997: 153-157, fig. 87, con un análisis de las influencias recíprocas que puede resultar muy revelador.

8 Sobre la cronología de los cascos en hierro vid. Mazzoli, 2016; García Jiménez y Graells, 2016; Graells, 2018.

9 Sievers, 1982, aunque las hojas suelen tender a una mayor anchura y a la forma convexa de su único filo, lo que las distingue del caso ligur que analizamos aquí. 
A estos paralelos pueden sumarse cuchillos de hierro de la fase Golasecca IIA de Lombardía y puñales, como el largo ejemplar de la tumba 3 de la necrópolis de Albenga (Massabò, Del Lucchese y Torre, 2014). La cronología de ambas series se fecha entre finales del s. VII y mediados del s. VI a. C., con lo que podría proponerse que la adopción de este tipo de filos únicos con refuerzo dorsal de la lámina iniciara primero con armas cortas y se intentara en armas más largas en un momento más tardío.

\section{CONCLUSIONES}

Los estudios sobre el armamento de las comunidades de la Edad del Hierro en el golfo de León han recibido en las últimas décadas una atención destacable que ha comportado una mejora considerable de su caracterización. Las abundantes publicaciones que han catalogado el repertorio iconográfico y los realia han revisado tanto las producciones locales como las importadas (Farnié y Quesada, 2005; Boyer, Dedet y Marchand, 2006; Beylier, 2012; Dedet y Marchand, 2015b; Graells, 2014; Garcia, Gruat y Marchand 2016; Farnié, 2016; Garcia, 2013). Especialmente destacadas son las tesis doctorales de A. Beylier (2012) y C. Farnié Lobensteiner (2013) que recopilan y estudian la práctica totalidad de armas prerromanas del golfo de León occidental y del norte de la península ibérica. Estos trabajos, además, se completan con los trabajos que han estudiado de manera intensiva algunas categorías de armas (normalmente defensivas) ${ }^{10}$ y particulares hallazgos individuales (tumbas o depósitos de armas) ${ }^{11}$. Se podría pensar que este panorama es satisfactorio y completo, especialmente si se compara con el conocimiento de finales del siglo pasado, pero aún hoy se echa en falta un estado de la cuestión sobre las armas del extremo oriental del golfo de León, principalmente de la región ligur ${ }^{12}$.

En cualquier caso, disponer de un conocimiento preciso de los tipos de armas usados y producidos en la mayor parte del golfo de León representa una aportación fundamental para el estudio de las comunidades que lo habitaron. Incluir las armas como interlocutor para el estudio de las transformaciones sociales y culturales del pasado supone un cambio, fruto a su vez de una renovación metodológica. Se han dejado de lado los análisis locales para evitar una lectura incompleta y se ha visto la necesidad de apostar por trabajos que permitan una lectura de las realidades arqueológicas desde perspectivas más amplias. Sorprendentemente, este tipo de investigaciones han supuesto un paso adelante en la investigación y han normalizado un modo de trabajo supranacional y supralingüístico que debería haberse superado hace mucho tiempo: que ni los límites políticos actuales ni la diversidad lingüística suponen ningún tipo de barrera que impida o dificulte la recopilación y discusión de los datos científicos ${ }^{13}$.

De este modo, desde una perspectiva amplia, podemos concluir que la proximidad de la espada en estudio con el ejemplar de Saint-Dalmas-de-Tende, por un lado, así como con

\footnotetext{
10 Cnémides (Farnié y Quesada, 2005) y corazas (Graells, 2014).

11 Tumbas: Dedet y Marchand, 2015b; Graells, 2015. - Depósitos de armas: Boyer, Dedet y Marchand, 2006. Otros: García Jiménez y Graells, 2016.

12 El trabajo de Beylier presenta un importante apéndice en el que se recopilan numerosas armas de esta región: Albegna (Beylier, 2012: 395); Chiavari (Beylier, 2012: 395-402); Génova (Beylier, 2012: 402); La Spezia (Beylier, 2012: 402); Pietra Ligura (Beylier, 2012: 402-404); Rapallo (Beylier, 2012: 404).

13 Aún, algunos estudios recientes han reiterado el viejo planteamiento aprovechando la presentación de un dossier de nuevos hallazgos iconográficos - caso de los discos coraza de la Francia meridional, recientemente publicados por D. García, P. Gruat y G. Marchand (2016) -. La crítica a este hecho no es tanto el no haber considerado propuestas y catálogos críticos expuestos en estudios recientes de mayor alcance (Graells, 2014) sino el no situar correctamente el problema y su discusión al desvincularlo de su posición espacial, intermedia y fundamental en relación a dinámicas de gran envergadura que relacionan la península italiana con la península ibérica y la Europa central.
} 
otros tipos de espada de un solo filo, permiten pensar en una producción ligur para la espada de Carcare. El ejemplar que aquí se presenta, y con toda probabilidad los otros ejemplares peor conservados, fueron el resultado de una fórmula experimental en la que se evidencia la apertura de la región ligur hacia influjos del Languedoc, las regiones centrales de Europa y la región balcánica. En efecto, la identificación de esta variante, hasta ahora inédita, en el extremo oriental del golfo de León aumenta la diversidad de tipos y tradiciones activas en esta región en la segunda mitad del s. VI y s. V a. C. pero subraya a su vez una necesidad: conocer en detalle el repertorio armamentístico de esta región para comprender el papel que tuvieron las comunidades ligures en la transferencia de modas y la combinación de influencias técnicas centroeuropeas, del golfo de León e itálicas.

\section{BIBLIOGRAFÍA}

Beylier, A. (2012): L'armement et le guerrier en Méditerranée nord-occidentale au premier âge du fer. Monographies d'Archéologie Méditerranéenne, 31. Lattes.

Boyer, R.; Dedet, B. y Marchand, G. (2006): «L'aven sépulcral de Plérimond à Aups, Var (VIe s. av. J.-C)». Gallia, 63: 171-209. https://doi.org/10.3406/galia.2006.3295

Dedet, B. y Marchand, G. (2015a): «Eau, arme et territoire aux Âges du Bronze final et du Fer en Languedoc et en Provence», Les Gaulois au fil de l'eau. Actes du 37e colloque international de l'Association Française pour l'étude de l'Âge du Fer (Montpellier, 2013). Bordeaux: 595-620.

Dedet, B. y Marchand, G. (2015b): «Héros, caciques et paysans armés en Languedoc et en Provence du VIIIe au IIe siècles avant J.-C.», Les estructures socials a la Gàl-lia i a Ibèria. Actes de la VII Reunió Internacional d'Arqueologia de Calafell (Calafell, 7 al 9 de marc de 2013). Arqueomediterrània, 14. Barcelona: 67-85.

Del Lucchese, A.; Maestro A. y Prestipino, C. (2015): «Pallare. Rio Cavallera. Spada ad antene». Archeologia in Liguria, n. s. V: 158-159.

Del Lucchese, A. y Prestipino, C. (2013): «Elmo di ferro rinvenuto in Località Rio Cavallera (Pallare)». Archeologia in Liguria, n. s. III: 129-130.

Dhennequin, L. (1999): «L'armement dans le Sud-Ouest de la France au début de l'Âge du Fer». Antiquités nationales, 31: 159-170.

Egg, M. (1992): «Ein eisenzeitlicher Altfund von Schloß Greifenstein bei Siebeneich in Südtirol», K. Spindler y A. Lippert (Hrsg.), Festschrift zum 50jährigen Bestehen des Institutes für Ur- und Frühgeschichte der Leopold Franzens Universität Innsbruck. Universtätsforschungen zur Prähistorischen Archäologie, 8. Bonn: 135-172.

Farnié, C. (2013): Las aristocracias de la primera Edad del Hierro en el Mediterráneo noroccidental: La espada como instrumento de guerra y símbolo de poder (ss. VIII-VI a.C.). Tesis doctoral no publicada, Univ. Autónoma de Madrid y Univ. Paris I 2013.

Farnié, C. (2016): «La influencia del armamento hallstático sobre el armamento de la Península Ibérica (Primera Edad del Hierro)», R. Graells y D. Marzoli (eds.), Armas de la Hispania Prerromana / Waffen im vorrömischen Hispanien. Actas del Encuentro Armamento y arqueología de la guerra en la Península Ibérica prerromana (s. VI-I a. C.): problemas, objetivos y estrategias / Akten der Tagung Bewaffnung und Archäologie des Krieges auf der Iberischen Halbinsel in vorrömischer Zeit (6.-1. Jh. v. Chr.): Probleme, Ziele und Strategien. RGZM-Tagungen 24. Mainz: 7-35.

Farnié, C. y Quesada, F. (2005): Espadas de hierro, grebas de bronce. Símbolos de poder e instrumentos de guerra a comienzos de la Edad del Hierro en la Península Ibérica. Monografías del Museo de Arte Ibérico de El Cigarralejo, 2. Murcia.

Gambari, F.M. (2000): «Il seppelimento rituale di elmi nei fiumi. Una pratica votiva dell'età del Ferro nella Cisalpina occidentale», L. Bonnamour (éd.), Archéologie des fleuves et des rivières. Paris: 204-208.

Garcia, D. (2013): «Le casque corinthien des Baux-de-Provence», S. Bouffier y A. Hermary (éds.), L'Occident grec de Marseille à Mégara Hyblaea. Hommages à Henri Tréziny. Bibliothèque d'Archéologie Méditerranéenne et Africaine, 13. Arles: 85-90. 
Garcia, D. ; Gruat, P. y Marchand, G. (2016): «Le cardiophylax, un élément de la panoplie du guerrier méridional du premier âge du Fer», Cl.-A. de Chazelles y M. Schwaller (éds.), Vie quotidienne, tombes et symboles des sociétés protohistoriques de Méditerranée nordoccidentale. Mélanges offerts à Bernard Dedet. Monographies d'Archéologie Méditerranéenne, Hors série, 7. Lattes: 721744.

García Jiménez, G. y Graells, R. (2016): «El trofeo de Can Miralles. el silo 24 y los trofeos de armas del nordeste de la península ibérica», Cl.-A. de Chazelles y M. Schwaller (éds.), Vie quotidienne, tombes et symboles des sociétés protohistoriques de Méditerranée nordoccidentale. Mélanges offerts à Bernard Dedet. Monographies d'Archéologie Méditerranéenne, Hors série n 7 . Lattes: 613-636.

Garibaldi, P. (2004): «Tomba di Roccatagliata (Genova)», R. C. De Marinis y G. Spadea (a cura di), I Liguri: un antico popolo europeo tra Alpi e Mediterraneo. Génova: 278-280.

Garibaldi, A. y Traverso, A. (2004): «Savignone (Genova)», R. C. De Marinis y G. Spadea (a cura di), I Liguri: un antico popolo europeo tra Alpi e Mediterraneo. Génova: 280-281.

Gaspari, A. y Mlinar, M. (2005): «Grave with a machaira from Most na Soci. Last versions of single-edged swords with a bent hilt». Arheoloski Vestnik, 56: 169-186.

Graells, R. (2014): «Discos-coraza de la Península Ibérica (s. VI-IV a.C.)». Jahrbuch des Römisch-Germanischen Zentralmuseums, 59: 85-244.

Graells, R. (2015): «El guerrero de Corno Lauzo: revisión de materiales», R. Roure (éd.), Contacts et acculturations en Méditerranée Occidentale. Hommages à Michel Bats (Hyères, 15-18 septembre 2011). Études Massaliètes, 12. Arles: 249-256.

Graells, R. (2018): «El casco de hierro de la tumba 27 de Galera». Cuadernos de Prehistoria y Arqueología de la Universidad de Granada, 27: 11-28.

Graells, R. y Lorrio, A. J. (2013): «El casco celtibérico de Muriel de la Fuente (Soria) y los hallazgos de cascos en las aguas en la Península Ibérica». Complutum, 24 (1): 151-173. https://doi.org/10.5209/ rev_cmpl.2013.v24.n1.42329

Graells, R. y Lorrio, A. J. (2016): «Helmets in the waters of the Iberian Peninsula: ritual practices and data for discussion», M. Egg, A. Naso y R. Röllinger (Hrgs.), Waffen für die Götter. Waffenweihungen in Archäologie und Geschichte, Akten der internationalen Tagung am Institut für Archäologien der Leopold-Franzens-Universität, Innsbruck, 6.-8. März 2013. RGZM-Tagungen 28. Mainz: 143-152.

Massabò, B.; Del Lucchese, A. y Torre, E. (2014): «La necropoli protostorica di Albenga (scavi 2004)». Antiche genti del Tigullio a Chiavari. Dalla necropoli ligure al Medioevo. Atti del Convegno di studi, Chiavari 24-25 settembre 2010 (Bordighera - Chiavari 2014): 129-137.

Mazzoli, M. (2016): «Elmi "Montefortino" nel Mediterraneo Occidentale», R. Graells y D. Marzoli (eds), Armas de la Hispania Prerromana / Waffen im vorrömischen Hispanien. Actas del Encuentro Armamento y arqueología de la guerra en la Península Ibérica prerromana (s. VI-I a. C.): problemas, objetivos y estrategias / Akten der Tagung Bewaffnung und Archäologie des Krieges auf der Iberischen Halbinsel in vorrömischer Zeit (6.-1. Jh. v. Chr.): Probleme, Ziele und Strategien. RGZM-Tagungen 24. Mainz: 109-147.

Mohen, J. P. (1980): L'Age du Fer en Aquitaine du VIIIe au IIIe s. av. J.-C., Mémoires de la Societé préhistorique Française, 14. París.

Paribeni, E. (2007): Guerrieri dell 'età del Ferro in Lunigiana. La Spezia.

Quesada, F. (1997): El armamento ibérico. Estudio tipológico, geográfico, funcional, social y simbólico de las armas en la Cultura Ibérica (siglos VI-I a.C.), vol. 1. Montagnac.

Sievers, S. (1982): Die mitteleuropäischen Hallstattdolche. Prähistorische Bronzefunde, VI, 6. München.

Recibido: 29-10-2018

Aceptado: 10-06-2019 